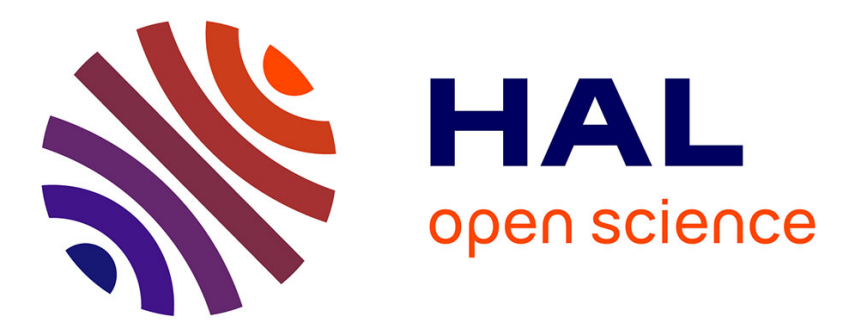

\title{
Matching and multiscale expansions for a model singular perturbation problem
}

Sébastien Tordeux, Grégory Vial, Monique Dauge

\section{To cite this version:}

Sébastien Tordeux, Grégory Vial, Monique Dauge. Matching and multiscale expansions for a model singular perturbation problem. Comptes Rendus. Mathématique, 2006, 343 (10), pp.637-642. 10.1016/j.crma.2006.10.010 . hal-00453366

\section{HAL Id: hal-00453366 https://hal.science/hal-00453366}

Submitted on 8 Dec 2017

HAL is a multi-disciplinary open access archive for the deposit and dissemination of scientific research documents, whether they are published or not. The documents may come from teaching and research institutions in France or abroad, or from public or private research centers.
L'archive ouverte pluridisciplinaire HAL, est destinée au dépôt et à la diffusion de documents scientifiques de niveau recherche, publiés ou non, émanant des établissements d'enseignement et de recherche français ou étrangers, des laboratoires publics ou privés. 


\title{
Matching and multiscale expansions for a model singular perturbation problem
}

\author{
Sébastien Tordeux ${ }^{\mathrm{a}}$, Grégory Vial ${ }^{\mathrm{b}}$, Monique Dauge $^{\mathrm{c}}$

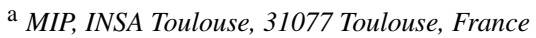 \\ b IRMAR, ENS Cachan Bretagne, 35170 Bruz, France \\ ${ }^{\mathrm{c}}$ IRMAR, Université de Rennes 1, 35042 Rennes, France
}

\begin{abstract}
We consider the Laplace-Dirichlet equation in a polygonal domain which is perturbed at the scale $\varepsilon$ near one of its vertices. We assume that this perturbation is self-similar, that is, derives from the same pattern for all values of $\varepsilon$. On the base of this model problem, we compare two different approaches: the method of matched asymptotic expansions and the method of multiscale expansion. We enlighten the specificities of both techniques, and show how to switch from one expansion to the other.
\end{abstract}

\section{Résumé}

Développements raccordé et multi-échelle pour un problème de perturbation singulière modèle. On considère le problème de Laplace-Dirichlet dans un domaine polygonal qui présente une perturbation de taille $\varepsilon$ en l'un de ses sommets. Cette perturbation est supposée auto-similaire, i.e. provient d'un motif fixe dilaté à l'échelle $\varepsilon$. Sur ce problème modèle, nous mettons en œuvre deux méthodes : développements asymptotiques raccordés et développement multi-échelle. Nous mettons en évidence les particularités de chaque approche et montrons comment passer d'un développement à l'autre.

\section{Version française abrégée}

On considère un polygone $\omega$ de $\mathbb{R}^{2}$, qu'on perturbe localement au voisinage d'un de ses sommets, $O$ placé à l'origine du repère. Le domaine $\omega$ coïncide au voisinage de $O$ avec un secteur infini $K$ dont l'angle associé est noté $\alpha$. La perturbation est auto-similaire : elle provient d'un motif $\Omega$ qui est un domaine infini coïncidant avec le même secteur $K$ à l'infini ; le domaine perturbé est défini comme :

$$
\omega_{\varepsilon}=\left\{x \in \omega ;|x|>\varepsilon R^{*}\right\} \cup\left\{x \in \varepsilon \Omega ;|x|<r^{*}\right\},
$$

E-mail addresses: sebastien.tordeux@insa-toulouse.fr (S. Tordeux), gvial@bretagne.ens-cachan.fr (G. Vial), monique.dauge@univ-rennes1.fr (M. Dauge). 
pour des $r^{*}$ et $R^{*}$ convenables, voir Fig. 1. Nous nous intéressons au problème modèle suivant :

Trouver $u_{\varepsilon} \in \mathrm{H}_{0}^{1}\left(\omega_{\varepsilon}\right) \quad$ tel que $\quad-\Delta u_{\varepsilon}=f \quad$ dans $\omega_{\varepsilon}$,

où le second membre $f \in \mathrm{L}^{2}\left(\omega_{\varepsilon}\right)$ a son support disjoint de la zone de perturbation.

Les singularités du problème de Dirichlet dans le secteur $K$ jouent un rôle essentiel dans la structure de $u_{\varepsilon}$. Rappelons qu'avec la notation $\lambda=\pi / \alpha$ et les coordonnées polaires $(r, \theta)$, ces singularités sont les $\mathfrak{s}^{p \lambda}=r^{p \lambda} \sin (p \lambda \theta)$ pour tout entier relatif $p[3,6]$.

La technique de développement asymptotique multi-échelle [7,1] consiste à construire une approximation globale de $u_{\varepsilon}$ dans $\omega_{\varepsilon}$, composée de deux types de termes : les premiers interviennent en la variable standard $x$ et les seconds en la variable dilatée $x / \varepsilon$. Les termes sont superposés à l'aide de fonctions de troncature ( $\chi$ est nulle proche du point $O, \psi$ est nulle à l'infini - voir (11) pour plus de détails) :

$$
u_{\varepsilon}(x)=\chi\left(\frac{x}{\varepsilon}\right) \sum_{\ell=0}^{n} \varepsilon^{\ell \lambda} v^{\ell \lambda}(x)+\psi(x) \sum_{\ell=0}^{n} \varepsilon^{\ell \lambda} V^{\ell \lambda}\left(\frac{x}{\varepsilon}\right)+\mathcal{O}\left(\varepsilon^{(n+1) \lambda}\right) .
$$

L'approche des développements asymptotiques raccordés [4,5] consiste à construire un développement intérieur, valide localement autour de la perturbation, et un autre, extérieur, valide seulement loin de la perturbation. Ils doivent être raccordés dans la région intermédiaire. On utilise une fonction de troncature $\varphi$ à l'échelle intermédiaire $r / \sqrt{\varepsilon}$ pour obtenir une approximation globale :

$$
u_{\varepsilon}(x)=\varphi\left(\frac{r}{\sqrt{\varepsilon}}\right) \sum_{\ell=0}^{n} \varepsilon^{\ell \lambda} u^{\ell \lambda}(r, \theta)+\left(1-\varphi\left(\frac{r}{\sqrt{\varepsilon}}\right)\right) \sum_{\ell=0}^{n} \varepsilon^{\ell \lambda} U^{\ell \lambda}\left(\frac{r}{\varepsilon}, \theta\right)+\mathcal{O}\left(\varepsilon^{(n+1) \lambda / 2}\right) .
$$

Chaque méthode a ses avantages et ses inconvénients, pour les principaux : la méthode multi-échelle présente l'avantage d'une approximation globale avec estimation optimale du reste, ainsi que le fait que tous les termes du développement sont solutions de problèmes variationnels, alors que la méthode de raccord permet de définir des termes intrinsèques, indépendamment de toute fonction de troncature. Par ailleurs, on peut passer de l'un à l'autre : il suffit de retrancher à $u^{n \lambda}$ ou à $U^{n \lambda}$ une combinaison linéaire de fonctions singulières duales $\mathfrak{s}^{-p \lambda}, p>0$, pour retrouver les termes variationnels $v^{n \lambda}$ ou $V^{n \lambda}$.

Les détails des preuves des résultats annoncés sont exposés dans [8].

\section{Introduction}

We consider families of self-similar perturbed domains defined thanks to two domains $\omega$ and $\Omega$ of $\mathbb{R}^{2}$, satisfying the following conditions for suitable positive numbers $r^{*}$ and $R^{*}$ :

- $\omega$ is a bounded polygon with one vertex at the origin $O$ : inside the ball centered in $O$ of radius $r^{*}, \omega$ coincides with an infinite plane sector $K$ of opening $\alpha$;

- $\Omega$ is an unbounded domain, coinciding with $K$ outside the ball centered in $O$ of radius $R^{*}$.

The domains $\omega_{\varepsilon}$ are defined as perturbations of $\omega$ using the pattern $\Omega$ : For $\varepsilon$ small enough

$$
\omega_{\varepsilon}=\left\{x \in \omega ;|x|>\varepsilon R^{*}\right\} \cup\left\{x \in \varepsilon \Omega ;|x|<r^{*}\right\} .
$$

Fig. 1 shows an example of such a situation: the corner is 'rounded' at vertex $O$ to the scale $\varepsilon$. Of course, our geometrical setting covers a wider range of cases, and the domain $\Omega$ may have corners itself, or even cracks. Besides, we do not need any assumption of inclusion of $\omega_{\varepsilon}$ into $\omega$ (or conversely). The domain $\omega_{\varepsilon}$ tends to $\omega$ as $\varepsilon$ goes to 0 , and $\Omega$ appears to be the limit of the family of domains $\varepsilon^{-1} \omega_{\varepsilon}$.

We denote by $u_{\varepsilon}$ the solution of the Laplace-Dirichlet equation in $\omega_{\varepsilon}$ :

$$
u_{\varepsilon} \in \mathrm{H}_{0}^{1}\left(\omega_{\varepsilon}\right) ; \quad-\Delta u_{\varepsilon}=f \quad \text { in } \omega_{\varepsilon},
$$

where $f$ is an $\mathrm{L}^{2}$-function whose support does not reach the origin $O$. The solution $u_{\varepsilon}$ clearly converges toward $u_{0} \in \mathrm{H}_{0}^{1}(\omega)$, satisfying $-\Delta u_{0}=f$ in $\omega$. We aim at describing precisely the asymptotic behavior of $u_{\varepsilon}$ by means of (i) a multi-scale expansion, and (ii) matched asymptotic expansions. We give in $\$ 2$ the first terms of these expansions, 

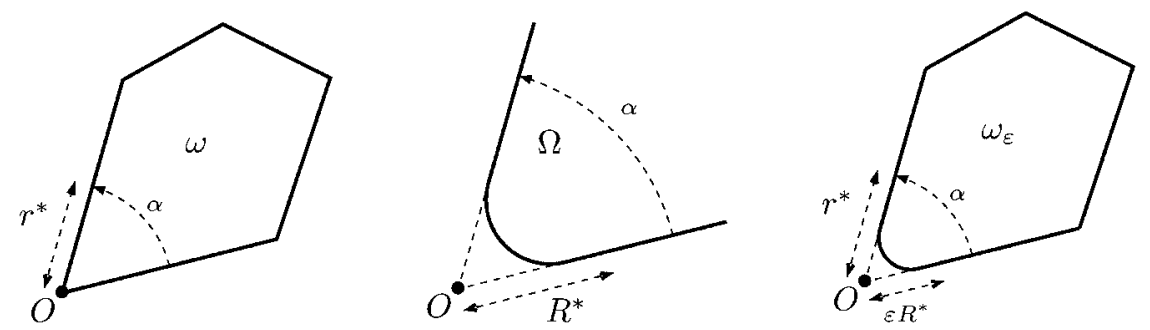

Fig. 1. Example of domains $\omega, \Omega$ and $\omega_{\varepsilon}$.

the complete expansions being described in $\S 3$ and $\S 4$, and compared with each other in $\S 5$. Details and proofs for the stated results can be found in [8].

The multi-scale approach of this problem has already been studied within a very general framework in [7]. Our purpose is to give for this model problem a simple explicit presentation for the two distinct, and even competing, techniques of multi-scale and matched expansions. Besides, this allows to prove formulas for transforming each expansion into the other one.

Notation. We need some notation used throughout the Note.

- Singular exponents and functions associated with the Laplace-Dirichlet problem in the sector $K$ are denoted by $p \lambda$ and $\mathfrak{s}^{p \lambda}$, respectively, with:

$$
\lambda=\frac{\pi}{\alpha}, \quad p \in \mathbb{Z}, p \neq 0 \quad \text { and } \quad \mathfrak{s}^{p \lambda}(r, \theta)=r^{p \lambda} \sin (\lambda \theta), \quad \text { with }(r, \theta) \text { polar coordinates in } K .
$$

- Remainders will be measured in the energy norm: we will write $\mathcal{O}_{\mathrm{H}^{1}}\left(\varepsilon^{\kappa}\right)$, which means that the norm in $\mathrm{H}^{1}\left(\omega_{\varepsilon}\right)$ is uniformly bounded by $C \varepsilon^{\kappa}$ as $\varepsilon \rightarrow 0$.

\section{The first terms of the expansions}

We present here the principles of the two methods, and provide the first terms of each expansion.

\subsection{Multi-Scale Expansion (MSE)}

The MSE method consists in looking for an expansion of $u_{\varepsilon}$ in powers of $\varepsilon$ with 'coefficients' combining the two scales $x$ and $\frac{x}{\varepsilon}$. Let $\chi$ be a smooth cut-off function vanishing at point $O$ and $\psi$ a smooth cut-off function localized near $O$. As a result of the MSE method we find for the first terms, see Theorem 1

$$
u_{\varepsilon}=\chi\left(\frac{x}{\varepsilon}\right) v^{0}(x)+\psi(x) \varepsilon^{\lambda} V^{\lambda}\left(\frac{x}{\varepsilon}\right)+\mathcal{O}_{\mathrm{H}^{1}}\left(\varepsilon^{2 \lambda}\right),
$$

and, next

$$
u_{\varepsilon}=\chi\left(\frac{x}{\varepsilon}\right)\left(v^{0}(x)+\varepsilon^{2 \lambda} v^{2 \lambda}(x)\right)+\psi(x)\left(\varepsilon^{\lambda} V^{\lambda}\left(\frac{x}{\varepsilon}\right)+\varepsilon^{2 \lambda} V^{2 \lambda}\left(\frac{x}{\varepsilon}\right)\right)+\mathcal{O}_{\mathrm{H}^{1}}\left(\varepsilon^{3 \lambda}\right) .
$$

Here, the first term $v^{0}$ coincides with the limit $u_{0}$. The profiles $V^{\lambda}$ and $V^{2 \lambda}$ are defined in the infinite pattern domain $\Omega$. Thus information concerning the perturbing pattern is contained in the profiles (whose contribution is localized near $O$ ), and $v^{0}, v^{2 \lambda}$ carry information corresponding to the bounded domain $\omega$ (whose influence does not reach the corner).

In our MSE analysis, all the terms $v(x)$ and all the profiles $V(X)$ are solution of variational problems in $\omega$ and $\Omega$, respectively. 


\subsection{Matched Asymptotic Expansions (MAEs)}

The MAEs method consists in constructing two full different expansions (outer and inner expansions, cf [4]) of $u_{\varepsilon}$ in powers of $\varepsilon$. The coefficients of the outer expansion are functions of the slow variable $x$, and those of the inner one are functions of the rapid variable $x / \varepsilon$. The outer expansion is valid far from $O$ and the inner one in a layer close to $O$. But neither of these two expansions is valid everywhere. They have to be matched and glued inside an intermediate region.

In order to have a representation of $u_{\varepsilon}$ everywhere and to optimize remainders, we use a cut-off function at the intermediate scale $r / \sqrt{\varepsilon}$. Let $\varphi$ be a smooth cut-off function with $\varphi(\rho)=0$ for $\rho \leqslant 1$ and $\varphi(\rho)=1$ for $\rho \geqslant 2$. As a result of the MAEs method we find for the first terms, see Theorem 2,

$$
u_{\varepsilon}=\varphi\left(\frac{r}{\sqrt{\varepsilon}}\right) u^{0}(x)+\left(1-\varphi\left(\frac{r}{\sqrt{\varepsilon}}\right)\right) \varepsilon^{\lambda} U^{\lambda}\left(\frac{x}{\varepsilon}\right)+\mathcal{O}_{\mathrm{H}^{1}}\left(\varepsilon^{\lambda}\right),
$$

and, next

$$
u_{\varepsilon}=\varphi\left(\frac{r}{\sqrt{\varepsilon}}\right)\left(u^{0}(x)+\varepsilon^{2 \lambda} u^{2 \lambda}(x)\right)+\left(1-\varphi\left(\frac{r}{\sqrt{\varepsilon}}\right)\right)\left(\varepsilon^{\lambda} U^{\lambda}\left(\frac{x}{\varepsilon}\right)+\varepsilon^{2 \lambda} U^{2 \lambda}\left(\frac{x}{\varepsilon}\right)\right)+\mathcal{O}_{\mathrm{H}^{1}}\left(\varepsilon^{3 \lambda / 2}\right) .
$$

Here, again, the first term $u^{0}$ coincides with the limit $u_{0}$. The term $u^{2 \lambda}$ is defined on $\omega$ whereas the profiles $U^{\lambda}$ and $U^{2 \lambda}$ are defined in the infinite domain $\Omega$. The terms $u^{\lambda}(x), U^{\lambda}(X)$ and $U^{2 \lambda}(X)$ are solution of 'super-variational problems', i.e. problems set in spaces larger than the variational spaces, and where standard formulations have nonunique solutions. Moreover, the terms $u^{p \lambda}$ and $U^{p \lambda}$ are, in general, distinct from the $v^{p \lambda}$ and $V^{p \lambda}$ and differ by a combination of dual singular functions, see Theorem 3 .

\section{Multiscale expansion}

In the MSE approach, the global expansion is valid everywhere and consists of terms involving the two scales $x$ and $x / \varepsilon$, superposed via cut-off functions: $\chi$ and $\psi$ are smooth and radial, satisfying:

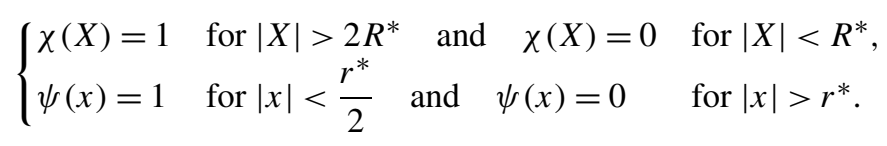

Theorem 1. The solution $u_{\varepsilon}$ of problem (6) admits the following multiscale expansion into powers of $\varepsilon$

$$
u_{\varepsilon}(x)=\chi\left(\frac{x}{\varepsilon}\right) \sum_{\ell=0}^{n} \varepsilon^{\ell \lambda} v^{\ell \lambda}(x)+\psi(x) \sum_{\ell=0}^{n} \varepsilon^{\ell \lambda} V^{\ell \lambda}\left(\frac{x}{\varepsilon}\right)+\mathcal{O}_{\mathrm{H}^{1}}\left(\varepsilon^{(n+1) \lambda}\right),
$$

where the terms $v^{\ell \lambda}$ and $V^{\ell \lambda}$ do not depend on $\varepsilon$, and are defined in $\omega$ and $\Omega$ by Eqs. (13) and (14).

\subsection{Short description of the terms}

Generically, $v^{\ell \lambda}$ and $V^{\ell \lambda}$ solve the variational problems

$$
\begin{aligned}
& v^{\ell \lambda} \in \mathrm{H}_{0}^{1}(\omega) \quad \text { such that } \forall v^{\prime} \in \mathrm{H}_{0}^{1}(\omega), \quad \int_{\omega} \nabla v^{\ell \lambda} \cdot \nabla v^{\prime}=\int_{\omega} f^{\ell \lambda} v^{\prime}, \\
& V^{\ell \lambda} \in \mathrm{W}_{0}^{1}(\Omega) \quad \text { such that } \forall V^{\prime} \in \mathrm{W}_{0}^{1}(\Omega), \quad \int_{\Omega} \nabla V^{\ell \lambda} \cdot \nabla V^{\prime}=\int_{\Omega} F^{\ell \lambda} V^{\prime},
\end{aligned}
$$

where $\mathrm{W}_{0}^{1}(\Omega)$ is the space $\left\{V \in \mathrm{W}^{1}(\Omega) ; V=0\right.$ on $\left.\partial \Omega\right\}$, with the weighted space $\mathrm{W}^{1}(\Omega)$ defined as:

$$
\mathrm{W}^{1}(\Omega)=\left\{V \in \mathcal{D}^{\prime}(\Omega) ;(1+R)^{-1} V \in \mathrm{L}^{2}(\Omega), \nabla V \in \mathrm{L}^{2}(\Omega)\right\} \quad \text { with } R=|X| .
$$

The right-hand sides $f^{\ell \lambda}$ and $F^{\ell \lambda}$ are defined recursively. 
- For $\ell=0$, we have $f^{0}=f$, defined in problem (6) ( $v^{0}$ is equal to the limit solution $\left.u_{0}\right)$, and $F^{0}=0$;

- For $\ell=1$, the function $f^{\lambda}$ is 0 and $F^{\lambda}=\mathbf{b}_{\lambda}^{0}\left(\left[\Delta_{X}, \chi\right] \mathfrak{s}^{\lambda}\right)=\mathbf{b}_{\lambda}^{0}\left(\Delta_{X}\left(\chi \mathfrak{s}^{\lambda}\right)-\chi \Delta_{X}\left(\mathfrak{s}^{\lambda}\right)\right)$, with the scalar coefficient $\mathbf{b}_{p \lambda}^{0}$ arising from the decomposition into singular functions of $v^{0}, \mathrm{cf}$. [6,3]:

$$
v^{0}(x)=\sum_{p=1}^{q} \mathbf{b}_{p \lambda}^{0} \mathfrak{s}^{p \lambda}(r, \theta)+\underset{r \rightarrow 0}{\mathcal{O}}\left(r^{q \lambda}\right), \quad \forall x \in \omega, \forall q \in \mathbb{N}
$$

- The next terms are generically nonzero: $f^{\ell \lambda}$ comes from the expansion at infinity of the previous profiles $V^{p \lambda}$ $(p<\ell)$, namely for the first one

$$
V^{\lambda}(X)=\sum_{p=1}^{q} \mathbf{B}_{p \lambda}^{\lambda} \mathfrak{s}^{-p \lambda}(R, \theta)+\underset{R \rightarrow \infty}{\mathcal{O}}\left(R^{-q \lambda}\right), \quad \forall X \in \Omega, \forall q \in \mathbb{N},
$$

and $F^{\ell \lambda}$ from the decomposition into singular functions of the previous terms $v^{p \lambda}(p<\ell)$.

\section{Matched asymptotic expansions}

Let $\varphi$ be a smooth cut-off with $\varphi(\rho)=0$ for $\rho \leqslant 1$ and $\varphi(\rho)=1$ for $\rho \geqslant 2$. The outer and inner expansions of $u_{\varepsilon}$ are glued together with the cut-off function $x \mapsto \varphi(r / \sqrt{\varepsilon})$ :

Theorem 2. The solution $u_{\varepsilon}$ of problem (6) admits the following expansion into powers of $\varepsilon$

$$
u_{\varepsilon}(x)=\varphi\left(\frac{r}{\sqrt{\varepsilon}}\right) \sum_{\ell=0}^{n} \varepsilon^{\ell \lambda} u^{\ell \lambda}(r, \theta)+\left(1-\varphi\left(\frac{r}{\sqrt{\varepsilon}}\right)\right) \sum_{\ell=0}^{n} \varepsilon^{\ell \lambda} U^{\ell \lambda}\left(\frac{r}{\varepsilon}, \theta\right)+\mathcal{O}_{\mathrm{H}^{1}}\left(\varepsilon^{(n+1) \lambda / 2}\right),
$$

where the terms $u^{\ell \lambda}$ and $U^{\ell \lambda}$ do not depend on $\varepsilon$, and are defined in $\omega$ and $\Omega$ by Eqs. (19) and (20).

\subsection{Short description of the terms}

Generically, $u^{\ell \lambda}$ and $U^{\ell \lambda}$ solve 'super-variational' problems:

Find $u^{\ell \lambda} \in V_{\mathrm{loc}, 0}(\omega) \quad$ such that $\quad \Delta u^{\ell \lambda}=-\delta_{0}^{\ell} f \quad$ in $\omega \quad$ and $\quad u^{\ell \lambda}-\sum_{p=1}^{\ell-1} a_{p \lambda}^{\ell \lambda} \mathfrak{s}^{-p \lambda} \in \mathrm{H}^{1}(\omega)$,

Find $U^{\ell \lambda} \in V_{\mathrm{loc}, \infty}(\Omega) \quad$ such that $\quad \Delta U^{\ell \lambda}=0 \quad$ in $\Omega \quad$ and $\quad U^{\ell \lambda}-\sum_{p=1}^{\ell} A_{p \lambda}^{\ell \lambda} \mathfrak{s}^{p \lambda} \in \mathrm{W}^{1}(\Omega)$,

where the spaces $V_{\mathrm{loc}, 0}(\omega)$ and $V_{\mathrm{loc}, \infty}(\Omega)$ are defined as

$$
\begin{aligned}
& V_{\mathrm{loc}, 0}(\omega)=\left\{u \in \mathcal{D}^{\prime}(\omega) ; \Phi u \in \mathrm{H}_{0}^{1}(\omega), \forall \Phi \in \mathcal{C}^{\infty}(\bar{\omega}) \text { with } O \notin \operatorname{supp}(\Phi)\right\} \\
& V_{\mathrm{loc}, \infty}(\Omega)=\left\{U \in \mathcal{D}^{\prime}(\Omega) ; \Phi U \in \mathrm{H}_{0}^{1}(\Omega), \forall \Phi \in \mathcal{C}^{\infty}(\bar{\Omega}) \text { with compact support }\right\}
\end{aligned}
$$

In (19) $\delta_{0}^{\ell}$ is the Kronecker symbol. The coefficients $a_{p \lambda}^{\ell \lambda}$ and $A_{p \lambda}^{\ell \lambda}$ are constructed by induction, and play also the role of right-hand sides: In these super-variational problems, uniqueness is enforced by prescribing an asymptotics in terms of dual singular functions. The coefficients $a_{p \lambda}^{\ell \lambda}$ and $A_{p \lambda}^{\ell \lambda}$ come from the decomposition into singular and dual-singular functions of the terms $U^{p \lambda}$ and $u^{p \lambda}$ for $p<\ell$, respectively.

Remark 1. The scale $r / \sqrt{\varepsilon}$ used in expansion (18) is, in a certain sense, arbitrary. If, instead, we choose the scale $x / \varepsilon^{\gamma}$ with $\gamma \in(0,1)$, we find for the remainder $\mathcal{O}_{\mathrm{H}^{1}}\left(\varepsilon^{(n+1) \lambda \min \{\gamma, 1-\gamma\}}\right)$. Thus the scale $r / \sqrt{\varepsilon}$ optimizes the remainder. 


\section{Comparison between the two expansions}

Theorem 3. The expansions (12) and (18) can be compared as follows: the terms $u^{n \lambda}$ and $v^{n \lambda}$ coincide away from the corner point i.e. for $r \geqslant r^{*}$; the profiles $U^{n \lambda}$ and $V^{n \lambda}$ coincide in the corner region i.e. for $R \leqslant R^{*} / 2$.

More precisely, we have the identities (for the definition of the coefficients $a_{p \lambda}^{n \lambda}$ and $A_{p \lambda}^{n \lambda}$, see Section 4)

$$
\begin{cases}v^{n \lambda}(x)=u^{n \lambda}(x)-\psi(x) \sum_{p=1}^{n-1} a_{p \lambda}^{n \lambda} \mathfrak{s}^{-p \lambda}(x), & x \in \omega, \\ V^{n \lambda}(X)=U^{n \lambda}(X)-\chi(X) \sum_{p=1}^{n} A_{p \lambda}^{n \lambda} \mathfrak{s}^{p \lambda}(X), & X \in \Omega .\end{cases}
$$

\section{Extensions}

Several extensions of the previous results are possible, requiring more or less effort. First, we can consider a smooth right-hand side $f$ whose support does reach the perturbation region. In this case, the so-called logarithmicpolynomial singularities have to be considered to take the Taylor expansion of $f$ near the corner point $O$ into account. Other boundary conditions may be treated as well. The Neumann case, for instance, is slightly more involved due to the logarithmic singularity and the absence of Poincaré inequality. Lastly, we mention the three-dimensional case: if the domain $\omega$ has only a conical point at $O$, the analysis is very similar to the two-dimensional case. However, in presence of edges, these techniques might still be used, but with non straightforward adaptations (see [2] for edge and corner-edge asymptotics without small parameters).

\section{References}

[1] G. Caloz, M. Costabel, M. Dauge, G. Vial, Asymptotic expansion of the solution of an interface problem in a polygonal domain with thin layer, Asymptotic Analysis (2006), in press.

[2] M. Dauge, Elliptic Boundary Value Problems in Corner Domains - Smoothness and Asymptotics of Solutions, Lecture Notes in Mathematics, vol. 1341, Springer-Verlag, Berlin, 1988.

[3] P. Grisvard, Boundary Value Problems in Non-Smooth Domains, Pitman, London, 1985.

[4] A. Il'lin, Matching of Asymptotic Expansions of Solutions of Boundary Value Problems, Translations of Mathematical Monographs, 1992.

[5] P. Joly, S. Tordeux, Matching of asymptotic expansions for wave propagation in media with thin slots I: The asymptotic expansion, Multiscale Modeling and Simulation 5 (1) (2006) 304-336.

[6] V.A. Kondrat'ev, Boundary value problems for elliptic equations in domains with conical or angular points, Trans. Moscow Math. Soc. 16 (1967) 227-313.

[7] V. Maz'ya, S.A. Nazarov, B.A. Plamenevskij, Asymptotic Theory of Elliptic Boundary Value Problems in Singularly Perturbed Domains, Birkhäuser, Berlin, 2000.

[8] S. Tordeux, G. Vial, M. Dauge, Selfsimilar perturbation near a corner: matching and multiscale expansions, 2006, in preparation. 